\title{
Yamada Polynomial and Crossing Number of Spatial Graphs
}

\author{
Tomoe MOTOHASHI, Yoshiyuki OHYAMA and Kouki TANIYAMA
}

\begin{abstract}
In this paper we estimate the crossing number of a flat vertex graph in 3-space in terms of the reduced degree of its Yamada polynomial.
\end{abstract}

\section{INTRODUCTION}

Throughout this paper we work in the piecewise linear category. For a graph $G$, we denote the set of all vertices of $G$ by $V(G)$ and the set of all edges of $G$ by $E(G)$. A graph $G$ in the 3-space $R^{3}$ is called a flat vertex graph if for each vertex $v$ of $G$, there exists a neighbourhood $B_{v}$ of $v$ and a small flat plane $P_{v}$ such that $G \cap B_{v} \subset P_{v}$. A flat deformation of $G$ is an ambient isotopy $h_{t}: R^{3} \rightarrow R^{3}, t \in[0,1], h_{0}=i d_{R^{3}}$ such that $h_{t}(G)$ is a flat vertex graph for each $t \in[0,1]$.

All graphs in this paper are flat vertex graphs. Two graphs $G_{1}$ and $G_{2}$ are said to be equivalent if there is a flat deformation $h_{t}: R^{3} \rightarrow R^{3}$ such that $h_{1}\left(G_{1}\right)=G_{2}$. From now on, we do not distinguish a graph and its equivalence class so long as no confusion occurs.

1991 Mathematics Subject Classification: 57M25, 57M15, 05C10

Editorial Complutense. Madrid, 1994. 
Let $\pi: R^{3} \rightarrow R^{2}$ be a projection defined by $\pi(x, y, z)=(x, y)$. If each multiple point of $\left.\pi\right|_{G}$ is a double point of two transversal edges of $G$, then we say that the image $\pi(G)$ is a regular projection of $G$ and denote it by $\widehat{G}$. A double point of $\widehat{G}$ is called a crossing point. We denote the number of crossing points in $\widehat{G}$ by $c(\widehat{G})$. The crossing number $c(G)$ of a graph $G$ is defined to be the minimal number of crossing points among all regular projections of $G$.

The crossing number of links has been well studied in terms of Jones polynomial and other polynomials [1] [2] [3] [4] [5] [6] [8] [9] [10] [11] [12] [13] [14] [15] [16] [18].

In this paper we study the crossing number of spatial graphs in terms of the reduced degree of Yamada polynomial defined in [17].

Kobayashi [7] studied the relation between the crossing number and Yamada polynomial but the results in [7] concern some special cases. We study it in a more general setting.

A regular projection $\widehat{G}$ together with over/under information at each crossing point of $\widehat{G}$ is called a diagram of $G$ and we denote it by $\tilde{G}$. By the one point compactification $R^{2} \cup\{\infty\}=S^{2}$, we consider that the regular projections and the diagrams are on the 2-sphere $S^{2}$.

A state $S$ of $\tilde{G}$ is a plane graph obtained from $\tilde{G}$ by replacing each crossing point of $\tilde{G}$ by one of the following spins in Fig. 1.1.

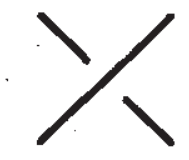

crossing point

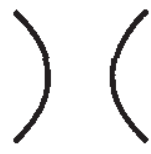

plus spin

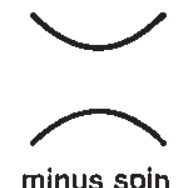

minus spin

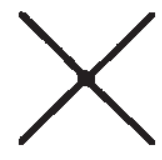

zero spin

Fig. 1.1

If $S$ contains a hoop then we add a vertex and make it a loop. See Fig. 1.2. 


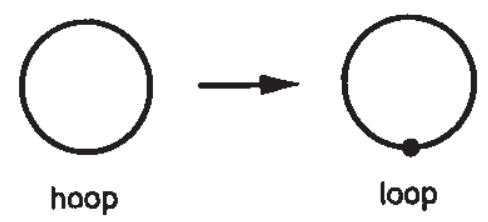

Fig. 1.2

Let $\mathbf{S}=\mathbf{S}(\tilde{G})$ be the set of all states of $\tilde{G}$. We have $|\mathbf{S}(G)|=3^{c(\tilde{G})}$ where || means the cardinality and $c(\tilde{G})=c(\hat{G})$.

Let $p(S)$ (resp. $m(S), z(S)$ ) be the number of plus (resp. minus, zero) spins in a state $S$. Let $A$ be a free variable and $\{\tilde{G} \mid S\}=$ $A^{p(S)-m(S)}$. Let

$$
H(S)=\sum_{F \subset E(S)}(-1)^{\beta_{0}(S-F)}\left(-A-2-A^{-1}\right)^{\beta_{1}(S-F)}
$$

where $F$ varies over all subsets of $E(S)$ and $S-F$ is a subgraph of $G$ defined by $V(S-F)=V(S)$ and $E(S-F)=E(S)-F$, and $\beta_{i}$ denotes the $i$-th Betti number.

Yamada polynomial $R_{\bar{G}}(A)$ is an element in the Laurent polynomial ring $Z\left[A, A^{-1}\right]$ defined by

$$
R_{\tilde{G}}(A)=\sum_{S \in \mathbf{S}}\{\tilde{G} \mid S\} H(S)
$$

where the summation is taken over all states of $\tilde{G}$.

Yamada proved in [17] that if $\bar{G}$ and $\bar{G}^{\prime}$ represent equivalent graphs then $R_{\tilde{G}}(A)=(-A)^{n} R_{\tilde{G}^{\prime}}(A)$ for some integer $n$. Let $\max (f(A)$ ) (resp. $\min (f(A)))$ be the maximal degree (resp. minimal degree) of a Laurent polynomial $f(A)$. Let $r(f(A))=\max (f(A))-\min (f(A))$ be the reduced degree of $f(A)$. We use the following convention: 
$\max (\tilde{G})=\max \left(R_{\tilde{G}}(A)\right), \min (\tilde{G})=\min \left(R_{\tilde{G}}(A)\right)$ and $r(\tilde{G})=$ $r\left(R_{\tilde{G}}(A)\right)$.

Then the reduced degree $r(G)$ of $G$ is well-defined by $r(G)=r(\bar{G})$.

We define a graph $\Gamma=\Gamma(\widehat{G})$ for a regular projection $\widehat{G}$ as follows:

(1) The vertices of $\Gamma$ correspond to the regions of $S^{2}-\widehat{G}$.

(2) For each crossing point $x$ of $\widehat{G}$, there are two edges of $\Gamma$ such that each of which joins the two regions that are incident at $x$.

See Fig. 1.3.

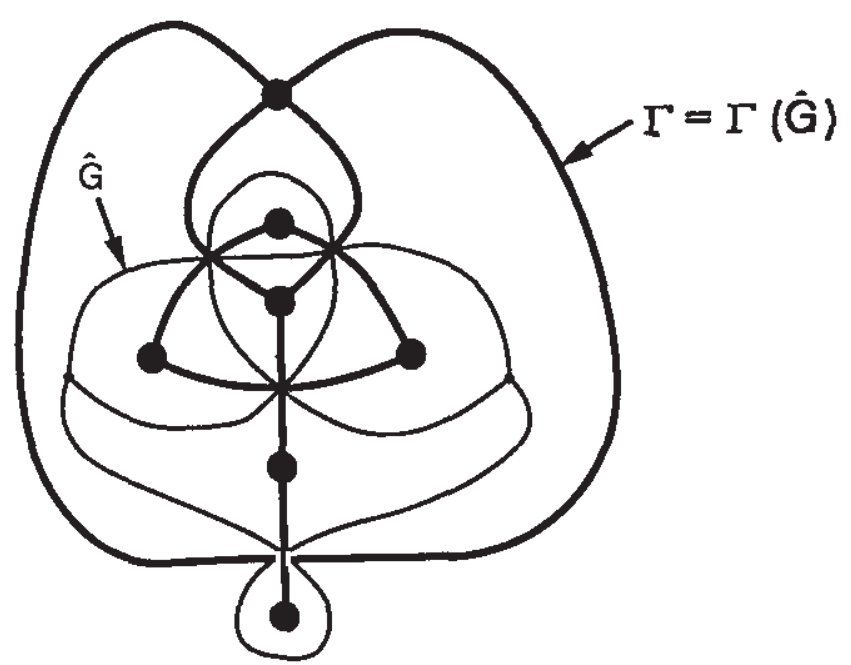

Fig. 1.3

Let $s(\widehat{G})=\beta_{0}(\Gamma(\widehat{G}))$ and let $s(G)$ be the maximum of $s(\widehat{G})$ among all regular projections of $G$. We will show in Proposition 1.3 that $s(G)$ always exists. We call $s(G)$ the splitting degree of $G$. 
The following is the main theorem of this paper.

Theorem 1.1. Let $G$ be a flat vertex graph in $R^{3}, \tilde{G}$ a diagram of $G$ and $\widehat{G}$ underlying projection of $\tilde{G}$. Then

$$
r(\tilde{G}) \leq 3 c(\tilde{G})-\chi(G)+s(\widehat{G})+\beta_{0}(\widehat{G})-1
$$

where $\chi(G)=\beta_{0}(G)-\beta_{1}(G)$ is the Euler characteristic of $G$.

Let $\nu(G)$ be the maximum of $\beta_{0}(\widehat{G})$ among all regular projections of $G$. Then the following is an immediate corollary.

\section{Corollary 1.2.}

$$
c(G) \geq \frac{1}{3}\{r(G)+\chi(G)-s(G)-v(G)+1\}
$$

We will show the following estimation of $s(G)$.

\section{Proposition 1.3.}

$$
s(G) \leq \beta_{1}(G)+1 .
$$

Therefore Corollary 1.2 implies:

\section{Corollary 1.4.}

$$
\begin{gathered}
c(G) \geq \frac{1}{3}\left\{r(G)+\chi(G)-\beta_{1}(G)-\nu(G)\right\}= \\
=\frac{1}{3}\left\{r(G)-2 \beta_{1}(G)+\beta_{0}(G)-\nu(G)\right\} .
\end{gathered}
$$

Since $\beta_{0}(G) \geq \nu(G)$ we have:

Corollary 1.5.

$$
c(G) \geq \frac{1}{3}\left\{r(G)-2 \beta_{1}(G)\right\}
$$


or equivalently,

$$
r(G) \leq 3 c(G)+2 \beta_{1}(G) .
$$

Kobayashi proved in [7] that if a planar graph $G$ has no cut edges and a diagram $\tilde{G}$ of $G$ is obtained from a diagram of $G$ without crossing points by adding alternating knot diagrams locally, then $r(\tilde{G})=3 c(\tilde{G})+$ $2 \beta_{1}(G)$. Therefore the inequalities in Corollary 1.5 are best possible.

As an example we consider Kinoshita's theta curve $\theta$ represented by a diagram $\tilde{\theta}$ of Fig. 1.4.

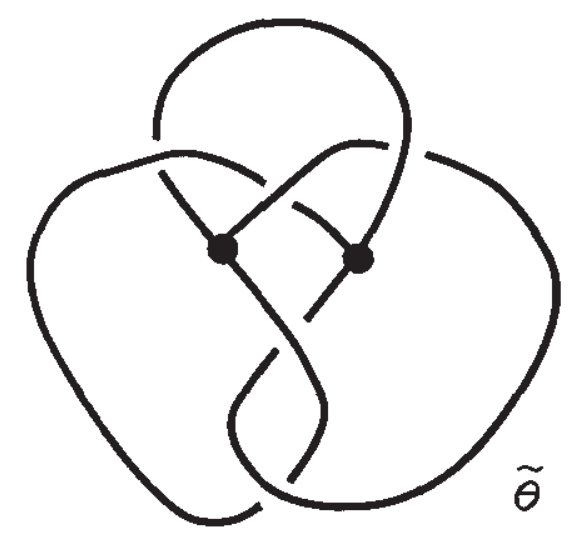

Fig. 1.4

Since $R_{\tilde{\theta}}(A)=A^{9}-A^{8}-2 A^{7}+A^{6}-A^{5}+2 A^{3}+A^{2}+2 A+A^{-1}-$ $A^{-3}+A^{-4}+A^{-5}-A^{-6}+A^{-7}+A^{-8}$ we have $r(\theta)=17$. Then by Corollary 1.5 we have $c(\theta) \geq \frac{1}{3}(17-2 \cdot 2)>4$. On the other hand $c(\theta) \leq c(\tilde{\theta})=5$. Thus we conclude $c(\theta)=5$.

We remark here that if the maximum valence of a graph $G$ is less than 4 , then the flat deformation equivalence coincides with the ambient isotopy equivalence, see [17], therefore $c(G)$ is minimal among all regular 
projections of $G$ up to ambient isotopy. In general, this is not the case. See Fig. 1.5 for an example.
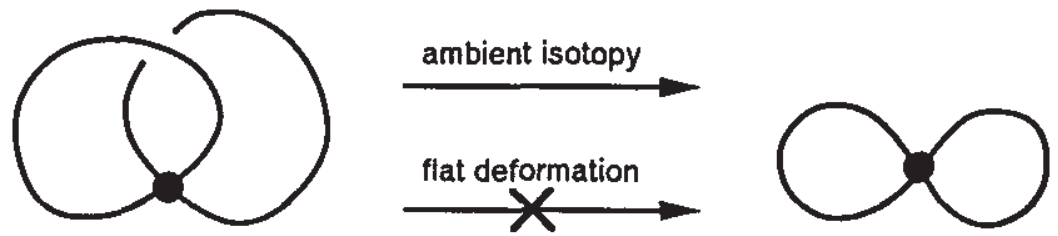

Fig. 1.5

This paper is organized as follows. In section 2 we prove Theorem 1.1 and Proposition 1.3. In section 3 we define adequate diagrams as a natural generalization of the adequate link diagrams defined in [8] and determine the reduced degree of the adequate diagrams. As a corollary, we show that adequate graphs have nonzero crossing numbers. In section 4 we define alternating diagrams and give an estimation of the crossing number of the graphs that have adequate alternating diagrams. In section 5 we give an estimation of $s(G)$ for some graphs which is sharper than that of Proposition 1.3 and determine their crossing numbers.

All of the authors are very grateful for Professor Shin'ichi Suzuki for his helpful advices and encouradgement.

\section{AN INEQUALITY}

Let $S_{+}=S_{+}(\tilde{G})$ be a state of $\tilde{G}$ characterized by $p\left(S_{+}\right)=c(\tilde{G})$ and $S_{-}=S_{-}(\tilde{G})$ characterized by $m\left(S_{-}\right)=c(\tilde{G})$. 


\section{Lemma 2.1.}

$$
\begin{gathered}
\max (\tilde{G}) \leq c(\tilde{G})+\beta_{1}\left(S_{+}(\tilde{G})\right) \\
\min (\tilde{G}) \geq-c(\tilde{G})-\beta_{1}\left(S_{-}(\tilde{G})\right)
\end{gathered}
$$

Sublemma 2.2. For any state $S$ of $\tilde{G}, \max (H(S)) \leq \beta_{1}(S)$ and $\min (H(S)) \geq-\beta_{1}(S)$.

Proof. By $(1.1)$ it is enough to show that $\beta_{1}(S-F) \leq \beta_{1}(S)$ for all subset $F$ of $E(S)$. But this is clear.

The following sublemma is a Yamada polynomial version of the corresponding result for the Jones polynomial [5]. The proof is also similar.

Sublemma 2.3. For any state $S$ of $\widehat{G}, \max (\{\tilde{G} \mid S\} H(S)) \leq \mathrm{c}(\tilde{G})+$ $\beta_{1}\left(S_{+}\right)$and $\min (\{\tilde{G} \mid S\} H(S)) \geq-c(\tilde{G})-\beta_{1}\left(S_{-}\right)$. Moreover, if $m(S) \neq$ 0 then $\max (\{\tilde{G} \mid S\} H(S)) \leq c(\tilde{G})+\beta_{1}\left(S_{+}\right)$, and if $p(S) \neq 0$ then $\min (\{\tilde{G} \mid S\} H(S))>-c(\tilde{G})-\beta_{1}\left(S_{-}\right)$.

Proof. We will show the inequalities about the maximal degree. We first note that $\max (\{\tilde{G} \mid S\} H(S))=\max \{\tilde{G} \mid S\}+\max H(S)=p(S)-$ $m(S)+\max H(S) \leq p(S)-m(S)+\beta_{1}(S)$ and that $p\left(S_{+}\right)-m\left(S_{+}\right)=$ $p\left(S_{+}\right)=c(\tilde{G})$.

Let $S_{p}, S_{m}$ and $S_{z}$ be a triple of states of $\tilde{G}$ which differ only at a crossing of $\tilde{G}$ where the spins are plus, minus and zero respectively. Then we have $\beta_{1}\left(S_{z}\right)-1 \leq \beta_{1}\left(S_{p}\right) \leq \beta_{1}\left(S_{z}\right)$ and $\beta_{1}\left(S_{z}\right)-1 \leq \beta_{1}\left(S_{m}\right) \leq \beta_{1}\left(S_{z}\right)$ by counting the Euler characteristics. Hence we have $\beta_{1}\left(S_{m}\right)-1 \leq$ $\beta_{1}\left(S_{p}\right) \leq \beta_{1}\left(S_{m}\right)+1$. On the other hand, $p\left(S_{m}\right)=p\left(S_{z}\right)=p\left(S_{p}\right)-1$ and $m\left(S_{p}\right)=m\left(S_{z}\right)=m\left(S_{m}\right)-1$. Therefore we can conclude that

$$
p\left(S_{z}\right)-m\left(S_{z}\right)+\beta_{1}\left(S_{z}\right) \leq p\left(S_{p}\right)-m\left(S_{p}\right)+\beta_{1}\left(S_{p}\right)
$$

and

$$
p\left(S_{m}\right)-m\left(S_{m}\right)+\beta_{1}\left(S_{m}\right)<p\left(S_{p}\right)-m\left(S_{p}\right)+\beta_{1}\left(S_{p}\right)
$$


For any state $S$ of $\tilde{G}$, there is a sequence of states of $\tilde{G} S_{1}=$ $S_{+}, S_{2}, S_{3}, \ldots, S_{n}=S$ such that $S_{i+1}$ is obtained from $S_{i}$ by replacing a plus spin of $S_{i}$ to a minus or zero spin. Therefore above argument ensures the conclusion.

The proof of Lemma 2.1 follows immediately from Sublemma 2.3.

The following is a graph version of the dual state lemma for links in [4] [5] [10] [15].

Lemma 2.4. Let $\tilde{G}$ be a diagram of $G$ and $\widehat{G}$ underlying projection of $\tilde{G}$. Then

$$
\beta_{1}\left(S_{+}\right)+\beta_{1}\left(S_{-}\right) \leq c(\tilde{G})-\chi(G)+s(\widehat{G})+\beta_{0}(\widehat{G})-1 .
$$

Proof. If $\widehat{G}=\widehat{G}_{1} \cup \cdots \cup \widehat{G}_{n}$ such that each $\widehat{G}_{i}$ is connected and $\widehat{G}_{i} \cap \widehat{G}_{j}=\emptyset$ for $i \neq j$, then $s(\widehat{G})=s\left(\widehat{G}_{1}\right)+\cdots+s\left(\widehat{G}_{n}\right)-n+1$. Therefore it is sufficient to show that $\beta_{1}\left(S_{+}\right)+\beta_{1}\left(S_{-}\right) \leq c(\hat{G})-\chi(G)+s(\widehat{G})$ for a connected projection $\widehat{G}$.

Since $\chi(\widehat{G})=\chi(G)-c(\widehat{G})$, we have $\beta_{1}(\widehat{G})=\beta_{0}(\widehat{G})-\chi(\widehat{G})=$ $1-\chi(G)+c(\widehat{G})$.

By Alexander duality on $S^{2}$, we have $|V(\Gamma)|=\beta_{1}(\widehat{G})+1$. Thus we have bigskip

$$
|V(\Gamma)|=2-\chi(G)+c(\widehat{G}) .
$$

Let $\Gamma_{+}$and $\Gamma_{-}$be the subgraphs of $\Gamma$ such that $V\left(\Gamma_{+}\right)=V\left(\Gamma_{-}\right)=$ $V(\Gamma), E\left(\Gamma_{+}\right) \cup E\left(\Gamma_{-}\right)=E(\Gamma), E\left(\Gamma_{+}\right) \cap E\left(\Gamma_{-}\right)=\emptyset$ and $\Gamma_{+} \cap S_{+}=$ $\emptyset, \Gamma-\cap S_{-}=\emptyset$. See Fig. 2.1 . 


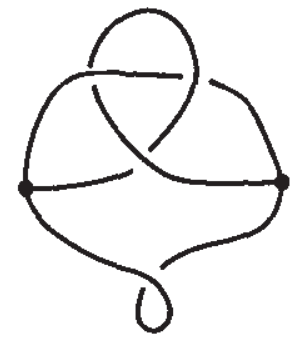

$\widetilde{\mathrm{G}}$

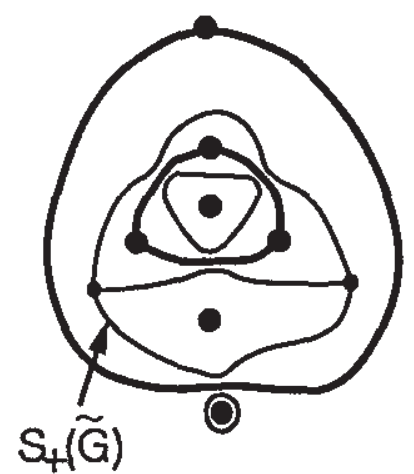

$\Gamma+(\widetilde{\mathbf{G}})$

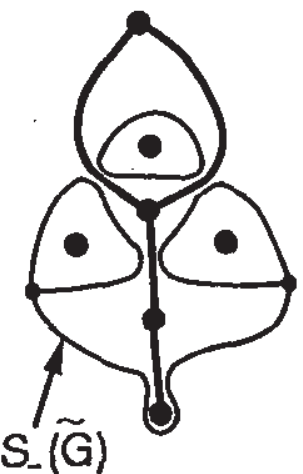

$\Gamma-(\widetilde{G})$

Fig. 2.1

By the Meyer-Vietoris sequence

$$
H_{0}(V(\Gamma)) \rightarrow H_{0}\left(\Gamma_{+}\right) \oplus H_{0}\left(\Gamma_{-}\right) \rightarrow H_{0}(\Gamma) \rightarrow 0
$$

we have

$$
\beta_{0}\left(\Gamma_{+}\right)+\beta_{0}\left(\Gamma_{-}\right) \leq \beta_{0}(\Gamma)+|V(\Gamma)| .
$$

By Alexander duality we have $\beta_{1}\left(S_{+}\right)=\beta_{0}\left(S^{2}-S_{+}\right)-1$ and $\beta_{1}\left(S_{-}\right)=\beta_{0}\left(S^{2}-S_{-}\right)-1$. From the fact that $S^{2}-\widehat{G}$ is a union of open 2-disks, it follows that $\Gamma_{+}$(resp. $\Gamma_{-}$) is a deformation retract of $S^{2}-S_{+}$(resp. $S^{2}-S_{-}$). Therefore we have

$$
\beta_{1}\left(S_{+}\right)=\beta_{0}\left(\Gamma_{+}\right)-1, \quad \beta_{1}\left(S_{-}\right)=\beta_{0}\left(\Gamma_{-}\right)-1 .
$$


By (2.1), (2.2) and (2.3) we have $\beta_{1}\left(S_{+}\right)+\beta_{1}\left(S_{-}\right)=\beta_{0}\left(\Gamma_{+}\right)+$ $\beta_{0}\left(\Gamma_{-}\right)-2 \leq \beta_{0}(\Gamma)+|V(\Gamma)|-2=c(\widehat{G})-\chi(G)+\beta_{0}(\Gamma)=c(\widehat{G})-\chi(G)+$ $s(\widehat{G})$.

Proof of Theorem 1.1. By Lemma 2.1 we have $r(\bar{G}) \leq 2 \mathfrak{c}(\tilde{G})+$ $\beta_{1}\left(S_{+}\right)+\beta_{1}\left(S_{-}\right)$. Then by Lemma 2.4 we have $r(\tilde{G}) \leq 3 c(\tilde{G})-\chi(G)+$ $s(\widehat{G})+\beta_{0}(\widehat{G})-1$.

Proof of Proposition 1.3. We will show the following claim by an induction on $c(G)$ :

Claim. If $\widehat{G}$ is a projection of a graph $G$, then $s(\widehat{G}) \leq \beta_{1}(G)+1$.

First suppose that $c(\widehat{G})=0$. Then $s(\widehat{G})=\beta_{0}(\Gamma(\widehat{G}))=|V(\Gamma(\widehat{G}))|$ and is equal to $\beta_{1}(\widehat{G})+1$ by Alexander duality. Thus $s(\widehat{G})=\beta_{1}(\widehat{G})+1=$ $\beta_{1}(G)+1$.

Next suppose that the claim is true when $c(\hat{G})=k$. Let $\hat{G}$ be a regular projection of $G$ with $c(\widehat{G})=k+1$. We choose an arbitrary crossing point $x$ of $\widehat{G}$. Then by smoothing at $x$ in two directions, we have new regular projections $\widehat{G}_{1}$ and $\widehat{G}_{2}$ of new graphs $G_{1}$ and $G_{2}$ respectively. See Fig. 2.2.

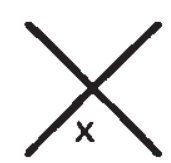

$\hat{\mathrm{G}}$

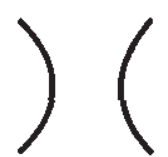

$\hat{G}$

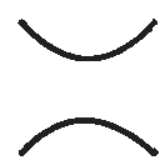

$\hat{\mathrm{G}_{2}}$

Fig. 2.2

We will show that at least one of $\beta_{1}\left(G_{1}\right)$ and $\beta_{1}\left(G_{2}\right)$ does not exceed $\beta_{1}(G)$. Since $\chi\left(G_{1}\right)=\chi\left(G_{2}\right)=\chi(G), \beta_{1}\left(G_{i}\right)>\beta(G)$ if and only if $\beta_{0}\left(G_{i}\right)>\beta_{0}(G), i=1,2$. 
Suppose that $\beta_{0}\left(G_{i}\right)>\beta(G), i=1,2$. Then the pair of smoothed arcs in $G_{i}$ lies in different components of $G_{i}, i=1,2$. But then the preimages of $x$ lie in different components in $G$. Therefore $\beta_{1}(G)=$ $\beta_{1}\left(G_{1}\right)=\beta_{1}\left(G_{2}\right)$, a contradiction. Thus we may suppose without loss of generality that $\beta_{1}\left(\dot{G}_{1}\right) \leq \beta_{1}(G)$.

It is easy to see that $\beta_{0}(\Gamma(\widehat{G})) \leq \beta_{0}\left(\Gamma\left(\widehat{G}_{1}\right)\right)$. See Fig. 2.3.
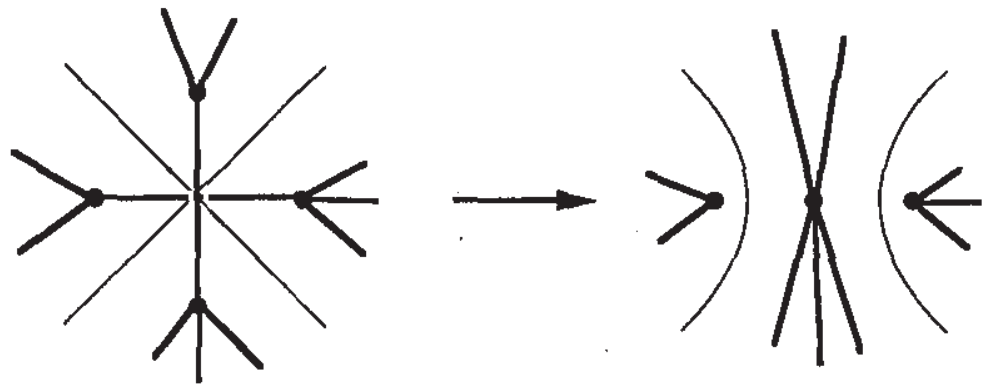

Fig. 2.3

By the hypothesis of induction, $s\left(\widehat{G}_{1}\right)=\beta_{0}\left(\Gamma\left(\hat{G}_{1}\right)\right) \leq \beta_{1}\left(G_{1}\right)+1$. Therefore $s(\widehat{G})=\beta_{0}(\Gamma(\widehat{G})) \leq \beta_{0}\left(\Gamma\left(\widehat{G}_{1}\right)\right) \leq \beta_{1}\left(G_{1}\right)+1 \leq \beta_{1}(G)+1$.

\section{ADEQUATE DIAGRAMS}

We intend to define adequate diagrams so that $r(\tilde{G})=2 c(\tilde{G})+$ $\beta_{1}\left(S_{+}(\tilde{G})\right)+\beta_{1}\left(S_{-}(\tilde{G})\right)$ for an adequate $\operatorname{diagram} \tilde{G}$.

An edge $e$ of a graph $S$ is called a cut edge if $\beta_{0}(S-\{e\})=\beta_{0}(S)+1$. We denote the set of all cut edges of $S$ by $C E(S)$.

Proposition 3.1. Let $S$ be a state. If $S$ has a cut edge, then $H(S)=0$. If $S$ has no cut edges, then $\max (H(S))=\beta_{1}(S)$ and $\min (H(S))=-\beta_{1}(S)$ and both of the coefficients of $A^{\beta_{1}(S)}$ and $A^{-\beta_{1}(S)}$ in $H(S)$ are $(-1)^{\beta_{0}(S)+\beta_{1}(S)}=(-1)^{x(S)}$. 
Above proposition in known in [17] and [7]. Therefore we give a sketch proof here.

Proof. Suppose that a state $S$ has a cut edge $e$. We divide the summation in (1.1) by whether or not $F$ contains $e$. then they cancel each other and we have $H(S)=0$.

Next suppose that $S$ has no cut edges. If $F$ is not empty, then $\beta_{1}(S-F)<\beta_{1}(S)$. Therefore the contribution for the maximal degree and the minimal degree by the term of $F=\emptyset$ does not disappear.

Let $S_{+}^{\prime}=S_{+}^{\prime}(\tilde{G})$ (resp. $S_{-}^{\prime}=S_{-}^{\prime}(\tilde{G})$ ) be a subdivision of $S_{+}=$ $S_{+}(\tilde{G})$ (resp. $S_{-}=S_{-}(\tilde{G})$ ) obtained by adding vertices at each spin as illustrated in Fig. 3.1.
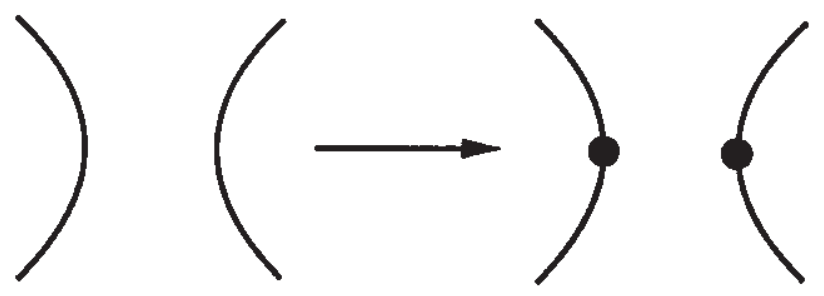

Fig. 3.1

Let $F_{+}=F_{+}(\bar{G})$ (resp. $F_{-}=F_{-}(\tilde{G})$ ) be a graph with $V\left(F_{+}\right)$(resp. $\left.V\left(F_{-}\right)\right)$corresponding to the connected components of $S_{+}^{\prime}-C E\left(S_{+}^{\prime}\right)$ (resp. $S_{-}^{\prime}-C E\left(S_{-}^{\prime}\right)$ ) and $E\left(F_{+}\right)=C E\left(S_{+}^{\prime}\right)\left(\right.$ resp. $E\left(F_{-}\right)=C E\left(S_{-}^{\prime}\right)$ ) with a natural incidence induced from that of $S_{+}^{\prime}$ (resp. $S_{-}^{\prime}$ ). We note that $\beta_{1}\left(F_{+}\right)=\beta_{1}\left(F_{-}\right)=0$.

Let $x$ be a crossing point of $\tilde{G}$. Let $S_{+, x}$ (resp. $S_{-, x}$ ) be the state obtained from $S_{+}$(resp. $S_{-}$) by replacing the plus (resp. minus) spin at $x$ to the zero spin. A crossing point $x$ is called plus essential (resp. minus essential) if $\beta_{1}\left(S_{+, x}\right)=\beta\left(S_{+}\right)+1$ (resp. $\beta_{1}\left(S_{-, x}\right)=\beta_{1}\left(S_{-}\right)+1$ ).

We may suppose that each plus (resp. minus) essential crossing point joins two vertices (that may be the same vertex) of $F_{+}$(resp. 
$F_{-}$). Let $J_{+}=J_{+}(\tilde{G})$ (resp. $J_{-}=J_{-}(\tilde{G})$ ) be a graph obtained from $F_{+}$(resp. $F_{-}$) by adding the edges that correspond to the plus (resp. minus) essential crossing points of $\tilde{G}$. See Fig. 3.2.

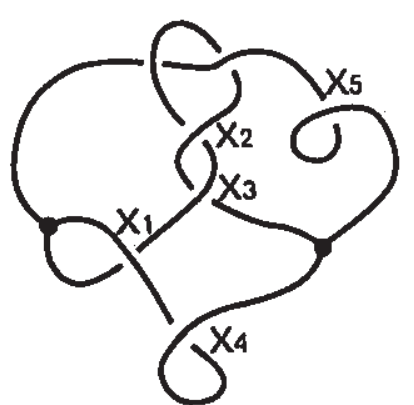

$\widetilde{\mathrm{G}}$

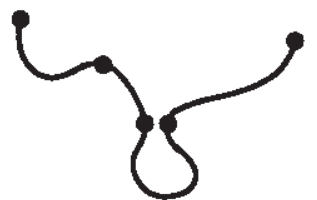

$\mathrm{F}+$

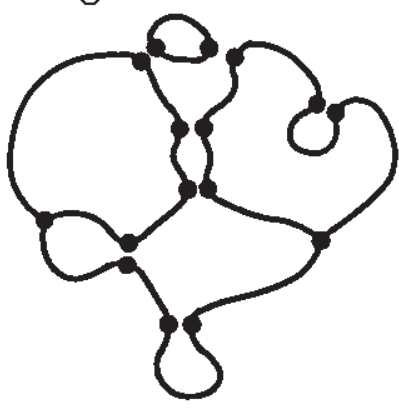

$S^{\prime}$

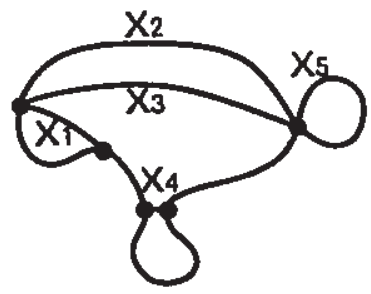

$J_{+}$

Fig. 3.2

Let $\rho_{k}$ (resp. $\mu_{k}$ ) be the number of subgraphs $K_{+}$(resp. $K_{-}$) of $J_{+}$(resp. $J_{-}$) with the following properties:

(1) $K_{+} \supset F_{+}$(resp. $\left.K_{-} \supset F_{-}\right)$.

(2) $K_{+}$(resp. $K_{-}$) has no cut edges.

(3) $\beta_{1}\left(K_{+}\right)=k\left(\right.$ resp. $\left.\beta_{1}\left(K_{-}\right)=k\right)$.

Definition 3.2. A diagram $\tilde{G}$ is plus adequate (resp. minus adequate) if $\sum_{k=0}^{\infty}(-1)^{k} \rho_{k} \neq 0$ (resp. $\sum_{k=0}^{\infty}(-1)^{k} \mu_{k} \neq 0$ ).

A diagram $\tilde{G}$ is adequate if $\tilde{G}$ is plus adequate and minus adequate. 
The diagram $\tilde{\theta}$ of Fig. 1.4 is an example of adequate diagram.

If $\tilde{G}$ is a link diagram, then $S_{+}^{\prime}$ (resp. $S_{-}^{\prime}$ ) has no cut edges. Therefore $F_{+}$(resp. $F_{-}$) has no edges and all edges of $J_{+}$(resp. $J_{-}$) are loops. Suppose that $J_{+}$(resp. $J_{-}$) has $n$ loops. Then it is clear that $\rho_{k}=\left(\begin{array}{l}n \\ k\end{array}\right)\left(\right.$ resp. $\left.\mu_{k}=\left(\begin{array}{l}n \\ k\end{array}\right)\right)$. By the binomial theorem we have $0=0^{n}=(1+(-1))^{n}=\sum_{k=0}^{n}(-1)^{k}\left(\begin{array}{l}n \\ k\end{array}\right)$. Therefore we have that $\tilde{G}$ is plus (resp. minus) adequate if and only if $n=0$. Since $n=0$ means that $\tilde{G}$ has no plus (resp. minus) essential crossings, $\tilde{G}$ is plus (resp. minus) adequate if and only if $\tilde{G}$ is a plus (resp. minus) adequate link diagram in the sense of [8].

\section{Theorem 3.3.}

(1) Let $\hat{G}$ be a plus adequate diagram. Then

$$
\max (\tilde{G})=c(\tilde{G})+\beta_{1}\left(S_{+}(\tilde{G})\right)
$$

and the coefficient of $A^{\max (\tilde{G})}$ in $R_{\tilde{G}}(A)$ is $(-1)^{x(G)} \sum_{k=0}^{\infty}(-1)^{k} \rho_{k}$.

(2) Let $\tilde{G}$ be a minus adequate diagram. Then

$$
\min (\tilde{G})=-c(\tilde{G})-\beta_{1}\left(S_{-}(\tilde{G})\right)
$$

and the coefficient of $A^{\min (\hat{G})}$ in $R_{\tilde{G}}(A)$ is $(-1)^{\chi(G)} \sum_{k=0}^{\infty}(-1)^{k} \mu_{k}$.

(3) Let $\tilde{G}$ be an adequate diagram. Then

$$
r(\tilde{G})=2 c(\tilde{G})+\beta_{1}\left(S_{+}(\tilde{G})\right)+\beta_{1}\left(S_{-}(\tilde{G})\right) .
$$

Proof. We will prove (1). Let $a_{i}(f(A))$ denote the coefficient of $A^{i}$ in a Laurent polynomial $f(A)$. By Lemma 2.1 it is sufficient to show that

$$
a_{c(\bar{G})+\beta_{1}\left(S_{+}(\bar{G})\right)}\left(R_{\bar{G}}(A)\right)=(-1)^{x(G)} \sum_{k=0}^{\infty}(-1)^{k} \rho_{k} .
$$


By Sublemma 2.3 and Proposition 3.1, it is sufficient to consider the states without minus spins and cut edges. Moreover, if a state $S$ has a zero spin at a crossing which is not plus essential, then we can show that $\max (\{\tilde{G} \mid S\} H(S))<\beta_{1}\left(S_{+}\right)+c(\tilde{G})$ by the argument in the proof of Sublemma 2.3.

Let $S$ be a state of $\tilde{G}$ without minus spins and cut edges such that each zero spin of $S$ occurs at a plus essential crossing point of $\tilde{G}$. Then it is easy to see that $\beta_{1}(S)=\beta_{1}\left(S_{+}\right)+z(S)$. Therefore $p(S)-m(S)+$ $\beta_{1}(S)=p(S)+\beta_{1}(S)=p(S)+\beta_{1}\left(S_{+}\right)+z(S)=c(\tilde{G})+\beta_{1}\left(S_{+}\right)$.

Then by Proposition 3.1 we have that

$$
\begin{gathered}
\max (\{\tilde{G} \mid S\} H(S))=\max \{\tilde{G} \mid S\}+\max (H(S))= \\
=p(S)+\beta_{1}(S)=c(\tilde{G})+\beta_{1}\left(S_{+}\right)
\end{gathered}
$$

and

$$
a_{c(\tilde{G})+\beta_{1}\left(S_{+}\right)}(\{\tilde{G} \mid S\} H(S))=(-1)^{x(S)}=(-1)^{x(G)+z(S)} .
$$

Let $K_{+}(S)$ be a subgraph of $J_{+}$which is obtained from $F_{+}$by adding the edges that correspond to the zero spins of $S$. Then $K_{+}(S)$ has no cut edges and $\beta_{1}\left(K_{+}(S)\right)=z(S)$. By this correspondence, we have that there are $\rho_{k}$ states of $G$ without minus spins and cut edges and with $k$ zero spins at plus essential crossing points. Their contribution to $a_{c(\bar{G})+\beta_{1}\left(S_{+}\right)}\left(R_{\bar{G}}(A)\right)$ is $(-1)^{\chi(G)+k} \rho_{k}$. Therefore we have

$$
a_{c(\tilde{G})+\beta_{1}\left(S_{+}\right)}\left(R_{\tilde{G}}(A)\right)=\sum_{k=0}^{\infty}(-1)^{\chi(G)+k} \rho_{k}=(-1)^{\chi(G)} \sum_{k=0}^{\infty}(-1)^{k} \rho_{k} .
$$

The following result corresponds to the result in link theory that adequate links are nontrivial [8].

Corollary 3.4. If a graph $G$ has an adequate diagram $\bar{G}$ with $c(\tilde{G})>0$, then $c(G)>0$ i.e. $G$ cannot be deformed into a plane $R^{2}$ in $R^{3}$ by any flat deformation. 
Proof. Let $\widehat{G}$ be the underlying projection of $\tilde{G}$. If $\beta_{1}(\widehat{G})=\beta_{1}(G)$, then we easily have that all states of $\tilde{G}$ has cut edges. Then $\tilde{G}$ is not adequate. Therefore we have that $\beta_{1}(\widehat{G})>\beta_{1}(G)$. Homologically, $\widehat{G}$ is obtained from $S_{+}(\tilde{G})$ (resp. $\left.S_{-}(\tilde{G})\right)$ by adding $c(\tilde{G})$ edges. Therefore we have $\beta_{1}\left(S_{+}(\tilde{G})\right)+c(\tilde{G}) \geq \beta_{1}(\widehat{G})$ and $\beta_{1}\left(S_{-}(\tilde{G})\right)+c(\tilde{G}) \geq \beta_{1}(\hat{G})$. Therefore, by Theorem $3.3(3)$ we have $r(G)=r(\tilde{G})=2 c(\tilde{G})+\beta_{1}\left(S_{+}(\tilde{G})\right)+$ $\beta_{1}\left(S_{-}(\tilde{G})\right) \geq 2 \beta_{1}(\hat{G})>2 \beta_{1}(G)$. Suppose that $G$ has a diagram without crossing points. Then we have $r(G) \leq 2 \beta_{1}(G)$ by Corollary 1.5 . This is a contradiction.

Examples of adequate diagrams are given in section 5 .

\section{ALTERNATING DIAGRAMS}

Definition 4.1. A diagram $\tilde{G}$ of a graph $G$ is weakly alternating if over crossing and under crossing appear alternately whenever we trace the image of an edge of $G$ from its end to its another end.

We remark here that this property is not topological. See Fig. 4.1.

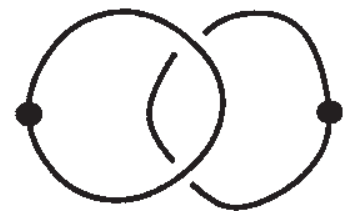

not weakly alţernating

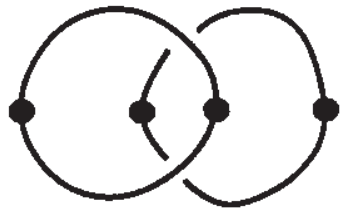

weakly alternating

Fig. 4.1

Let $\widehat{G}$ be a regular projection of a graph $G$. If we forget the position of $G$ in $R^{3}$ and consider $G$ as an abstract graph, then $\widehat{G}$ is called a drawing of $G$. For a drawing $\widehat{G}$, there are $2^{c(\widehat{G})}$ diagrams that have $\widehat{G}$ as their common underlying projection. We denote the set of these diagrams by $D(\widehat{G})$.

A drawing $\widehat{G}$ is said to be alternatable if $D(\widehat{G})$ contains an weakly alternating diagram. 
A graph $G$ is said to be Eulerian if $G$ has no odd-valence vertices.

Theorem 4.2. A drawing $\widehat{G}$ of a graph $G$ is alternatable if and only if we can obtain a drawing $\widehat{G}^{\prime}$ of an Eulerian graph $G^{\prime}$ from $\widehat{G}$ by adding edges to $\widehat{G}$ without introducing new crossing points and vertices.

Proof. If $\hat{G}^{\prime}$ is a drawing of an Eulerian graph $G^{\prime}$, then it is easy to see that $\hat{G}^{\prime}$ admits a checkerboard coloring by two colors, say black and white. We give over/under information at each crossing point of $\widehat{G}^{\prime}$ as illustrated in Fig. 4.2.

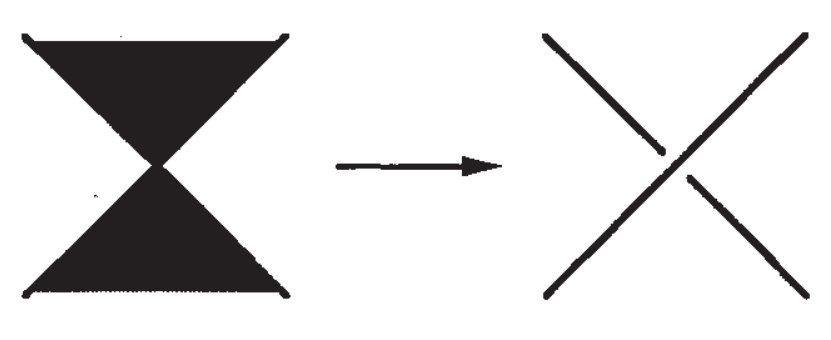

Fig. 4.2

Then the resultant diagram $\tilde{G}^{\prime}$ is weakly alternating. Therefore $\hat{G}^{\prime}$ is alternatable and therefore $\widehat{G}$ is also alternatable.

Next we will show the converse. Suppose that we cannot obtain any drawing of an Eulerian graph from $\widehat{G}$ without introducing new crossings and vertices. Let $\widehat{G}^{\prime}$ be a drawing of a graph $G^{\prime}$ obtained from $\widehat{G}$ without introducing new crossings and vertices such that the number of oddvalence vertices of $G^{\prime}$ is minimal among all such drawings.

Let $v$ 'be an odd-valence vertex of $\widehat{G}^{\prime}$ and let $R_{v}$ be the component of $\left(S^{2}-\widehat{G}^{\prime}\right) \cup V\left(\hat{G}^{\prime}\right)$ that contains $v$. If $R_{v}$ contains another odd-valence vertex $w$, the we choose a simple path from $v$ to $w$ in $R_{v}$. This path can be realized by some edges that are attached to $\hat{G}^{\prime}$. This contradicts to the minimality of the number of odd-valence vertices of $G^{\prime}$. Therefore 
we have that $R_{v}$ contains no other odd-valence vertices. The boundary cicuits of $R_{v}$ contains four types of crossings as illustrated in Fig. 4.3.

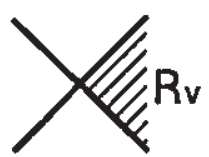

type I

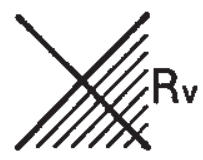

type II

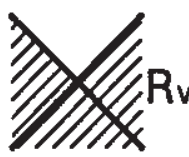

type III

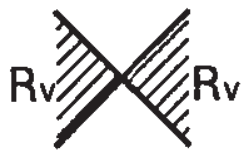

type IV

Fig. 4.3

By the handshaking lemma of graph theory we have that the number of type II crossing points is odd. Then it follows easily that at least one of the boundary circuits of $R_{v}$ cannot admit alternating over/under crossing information. Therefore $\widehat{G}^{\prime}$ and $\widehat{G}$ are not alternatable.

An example of non-alternatable drawing is illustrated in Fig. 4.4.

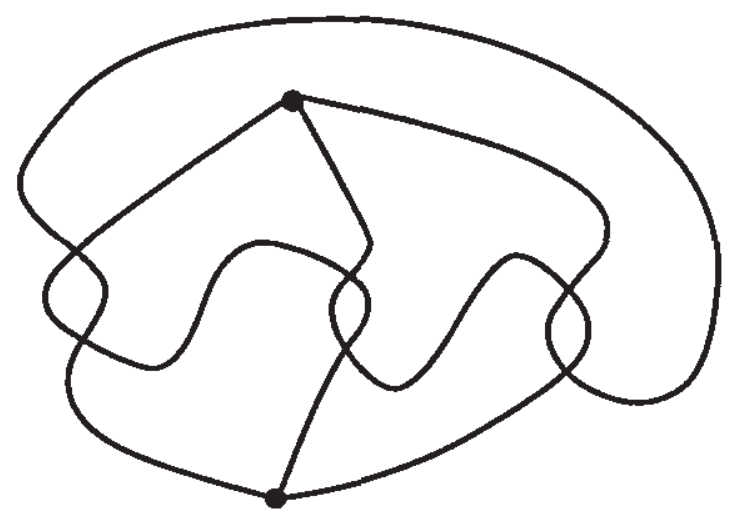

Fig. 4.4 
A diagram $\tilde{G}$ is said to be connected if its underlying projection is connected.

Let $o(G)$ be the number of odd-valence vertices of a graph $G$. We remark here that $o(G)$ is always even.

Definition 4.3. A connected diagram $\tilde{G}$ of a graph $G$ is alternating if there is a diagram $\tilde{G}^{\prime}$ of an Eulerian graph $G^{\prime}$ obtained from $\tilde{G}$ by adding $\frac{o(G)}{2}$ edges without introducing new crossing points and vertices such that the over/under crossing information of $\tilde{G}$ comes from a checkerboard coloring of $\tilde{G}^{\prime}$ as in Fig. 4.2.

$A$ diagram $\tilde{G}$ is alternating if each connected component of $\tilde{G}$ is alternating.

The diagram $\tilde{\theta}$ of Fig. 1.4 is an example of alternating diagram.

Theorem 4.4. Let $\tilde{G}$ be an alternating diagram of a graph $G$ and $\widehat{G}$ its underlying projection. Then

$$
\beta_{1}\left(S_{+}(\tilde{G})\right)+\beta_{1}\left(S_{-}(\tilde{G})\right) \geq c(\tilde{G})+2 \beta_{0}(\widehat{G})-\chi(G)-\frac{o(G)}{2} .
$$

Proof. It is sufficient to show

$$
\beta_{1}\left(S_{+}(\tilde{G})\right)+\beta_{1}\left(S_{-}(\tilde{G})\right) \geq c(\tilde{G})+2-\chi(G)-\frac{o(G)}{2}
$$

for a connected alternating diagram $\tilde{G}$ of a graph $G$.

Let $\tilde{G}^{\prime}$ be a diagram of an Eulerian graph $G^{\prime}$ obtained from $\tilde{G}$ by adding $\frac{o(G)}{2}$ edges such that the over/under crossing information of $\tilde{G}^{\prime}$ (and hence $\tilde{G}$ ) comes from a checkerboard coloring of $\hat{G}^{\prime}$. Then it is clear that $\beta_{1}\left(S_{+}\left(\tilde{G}^{\prime}\right)\right)$ (resp. $\beta_{1}\left(S_{-}(\tilde{G})\right)$ ) is greater than or equal to the number of white (resp. black) regions. It is easy to see that the number of regions of $\left(S^{2}, \hat{G}^{\prime}\right)$ is equal to $c\left(\tilde{G}^{\prime}\right)-\chi\left(G^{\prime}\right)+2$. Since $c\left(\tilde{G}^{\prime}\right)=c(\tilde{G})$ and $\chi\left(G^{\prime}\right)=\chi(G)-\frac{o(G)}{2}$ we have

$$
\beta_{1}\left(S_{+}\left(\tilde{G}^{\prime}\right)\right)+\beta_{1}\left(S_{-}\left(\tilde{G}^{\prime}\right)\right) \geq c(\tilde{G})+2-\chi(G)+\frac{o(G)}{2} .
$$


It is clear that $\beta_{1}\left(S_{+}(\tilde{G})\right) \geq \beta_{1}\left(S_{+}\left(\tilde{G}^{\prime}\right)\right)-\frac{\mathrm{o}(G)}{2}$ and $\beta_{1}(S-(\tilde{G})) \geq$ $\beta_{1}\left(S-\left(\tilde{G}^{\prime}\right)\right)-\frac{o(G)}{2}$. Therefore we have the conclusion.

If $\tilde{G}=\tilde{\theta}$ of Fig. 1.4, then we have $\beta_{1}\left(S_{+}(\tilde{G})\right)=4$ and $\beta_{1}\left(S_{-}(\tilde{G})\right)=$ 3. Therefore the inequality of Theorem 4.4 is best possible.

The following corollary follows immediately from Theorem $3.3(3)$ and Theorem 4.4 .

Corollary 4.5. Let $\tilde{G}$ be an adequate alternating diagram of a graph $G$ and $G$ its underlying projection. Then

$$
r(G) \geq 3 c(\tilde{G})-\chi(G)+2 \beta_{0}(\widehat{G})-\frac{o(G)}{2} .
$$

Corollary 4.6. Let $\tilde{G}$ be an adequate alternating diagram of a graph $G$ and $G$ its underlying projection. Then

$$
c(\tilde{G})-c(G) \leq \frac{1}{3}\left\{s(G)+\nu(G)+\frac{o(G)}{2}-2 \beta_{0}(\widehat{G})-1\right\} .
$$

Proof. By Corollary 4.5 and Corollary 1.2 , we have

$$
3 c(\tilde{G})-\chi(G)+2 \beta_{0}(\widehat{G})-\frac{o(G)}{2} \leq r(G) \leq 3 c(G)-\chi(G)+s(G)+\nu(G)-1
$$

Therefore we have the result.

Corollary 4.7. If $\tilde{G}$ is an adequate alternating diagnam of a nonsplittable graph $G$, then

$$
c(\tilde{G})-c(G) \leq \frac{1}{3}\left\{s(G)+\frac{o(G)}{2}-2\right\} .
$$

Therefore if $s(G) \leq 4-\frac{o(G)}{2}$, then $c(\tilde{G})=c(G)$. 
Remark. It is well known that a reduced alternating link diagram is adequate. But there are many non-adequate alternating diagrams. See Fig. 4.5 for such an example.

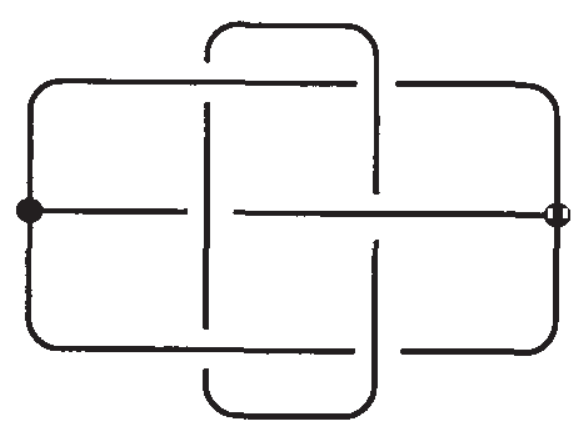

Fig. 4.5

\section{EXAMPLES}

In this section we show some examples of diagrams with minimal number of crossings.

Some more general statements can be obtained by the same method in this section. But we only consider some restricted cases for the simplicity.

It seems to the authors that one of the difficulties of deciding crossing numbers of spatial graphs is the difficulty of the estimation of the splitting degree $s(G)$.

Proposition 5.1. Let $G$ be a link with $\nu(G)=n$. Then $s(G)=$ $n+1$.

Proof. If $\widehat{G}$ is a connected link projection, then we easily have $s(\widehat{G})=\beta_{0}(\Gamma(\widehat{G}))=2$ because $\widehat{G}$ admits a checkerboard coloring. Therefore we have the result. 
Corollary 5.2. [10][12][6] $c(G)$.

If $\bar{G}$ is a reduced alternating link diagram of a link $G$, then $c(\tilde{G})=$

Proof. Let $\widehat{G}$ be the underlying projection of $\tilde{G}$. Let $\nu(G)=n$ and $\beta_{0}(\widehat{G})=m$. Then $m \leq n$. Suppose that $m<n$. Then there is a diagram $\tilde{G}^{\prime}$ of $G$ such that $c\left(\tilde{G}^{\prime}\right) \leq c(\tilde{G})-2(n-m)$ and $\beta_{0}\left(\hat{G}^{\prime}\right)=n$ where $\widehat{G}^{\prime}$ is the underlying porjection of $\tilde{G}^{\prime}$. A reduced alternating link diagram is adequate. Therefore by Corollary 4.5 we have $r(G) \geq 3 c(\tilde{G})+2 m$. On the other hand, by Theorem 1.1 and Proposition 5.1 we have $r(G) \leq$ $3 c\left(\tilde{G}^{\prime}\right)+2 n \leq 3 c(\tilde{G})-4 n+6 m$. Therefore we have $m \geq n$. Thus we have $m=n$.

Then by Corollary 4.6 and Proposition 5.1 we have

$$
c(\tilde{G})-c(G) \leq \frac{1}{3}\{n+1+n+0-2 n-1\}=0 .
$$

A theta curve $G$ in $R^{3}$ is said to be globally knotted if $G$ is not obtained from an unknotted theta curve $G_{0} \subset R^{2} \subset R^{3}$ by local sum of knots.

Proposition 5.3. Let $G$ be a globally knotted theta curve. Then $s(G)$ is less than or equal to two.

Proof. We use the fact that if a projection $\widehat{G}_{2}$ is obtained from a projection $\widehat{G}_{1}$ by smoothing a crossing point of $\widehat{G}_{1}$, then $s\left(\widehat{G}_{1}\right) \leq s\left(\widehat{G}_{2}\right)$, cf. Fig. 2.3.

Let $\widehat{G}$ be a projection of $G$. Then $\widehat{G}$ necessarily have a crossing point of different edges. We will obtain the projection $\widehat{G}_{0}$ of Fig. 5.1 by a series of smoothing of $\widehat{G}$. Then we have $s(\widehat{G}) \leq s\left(\widehat{G}_{0}\right)=2$. 


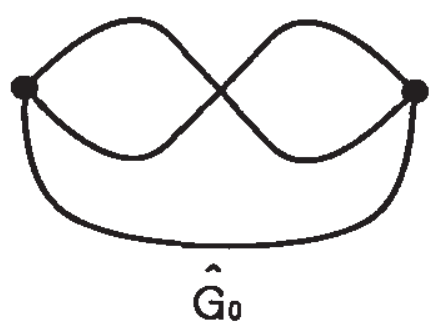

Fig. 5.1

We can eliminate by smoothing all crossings each of which is of one edge. See Fig. 5.2.

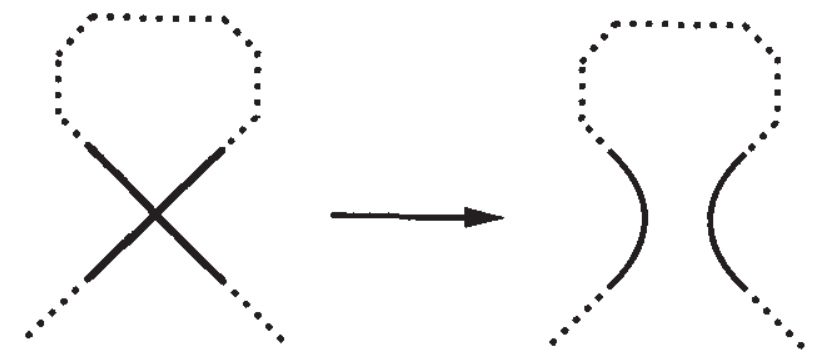

Fig. 5.2

Thus we may suppose that each crossing point of $\widehat{G}=\widehat{e}_{1} \cup \widehat{e}_{2} \cup \widehat{e}_{3}$ are of $\widehat{e}_{i}$ and $\widehat{e}_{j}$ for some $i \neq j$, where $\widehat{e}_{i}$ is the image of an edge $e_{i}$ of $G$. Let $V(G)=\left\{v_{1}, v_{2}\right\}$ and $\widehat{v}_{i}$ the image of $v_{i}$. We may suppose without loss of generality that $\widehat{e}_{1}$ has crossing points and the first crossing point $c_{0}$ which we encounter when we trace $\widehat{e}_{1}$ from $\widehat{v}_{1}$ is of $\widehat{e}_{1}$ and $\widehat{e}_{2}$. Then the arcs from $\widehat{v}_{1}$ to $c_{0}$ bounds a disk $\delta$ on $S^{2}$. By smoothing and renumbering 
of edges if necessary, we may suppose that $\widehat{e}_{3}$ runs away from $\delta$ at $\widehat{v}_{1}$ as illustrated in Fig. 5.3.

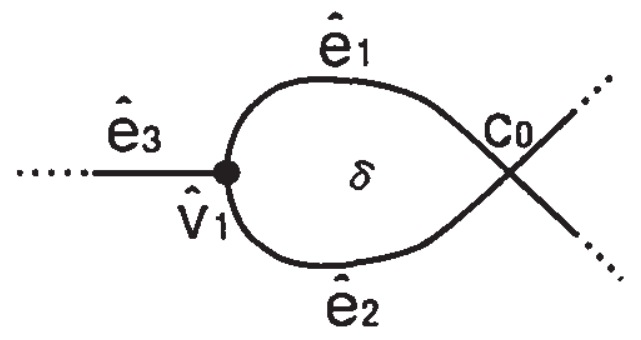

Fig. 5.3

By a series of smoothings of Fig. 5.4 and the innermost argument, we have that $\partial \delta$ has no crossing points except $c_{0}$.

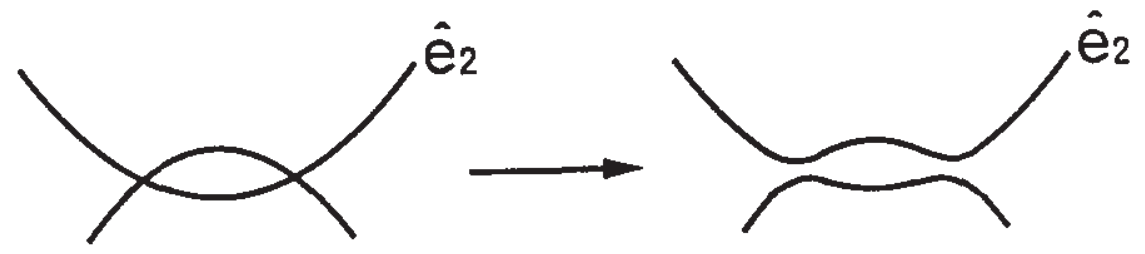

Fig. 5.4

One of the two smoothings at a crossing point preserves that $\widehat{G}$ is a projection of a theta curve. Therefore we can eliminate all the other crossing points and obtain $\widehat{G}_{0}$. 
Let $\tilde{G}$ be a diagram of a theta curve $G$ and $\widehat{G}$ its underlying projection.

Definition 5.4. A projection $\widehat{G}$ of a theta curve is reduced if $\widehat{G}$ has no crossings of the types illustrated in Fig. 5.5 where $T_{i}$ is any tangle possibly with vertices in it.

A diagram $\tilde{G}$ of a theta curve is reduced if its underlying projection is reduced.

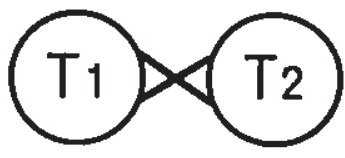

(a)

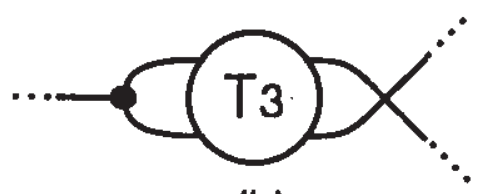

(b)

Fig. 5.5

If $\tilde{G}$ is alternating, then the vertices of $\hat{G}$ lie on the boundary of a region of $S^{2}-\widehat{G}$.

Theorem 5.5. Let $\tilde{G}$ be a reduced alternating diagram of a theta curve $G$ in $R^{3}$. If $\tilde{G}$ has the local part illustrated in Fig. 5.6, then $\tilde{G}$ is adequate and $c(\tilde{G})=c(G)$. 


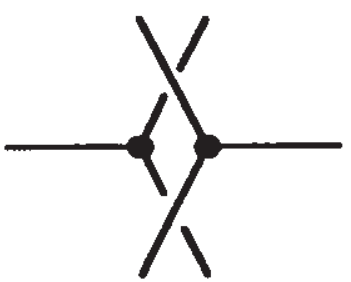

Fig. 5.6

Proof. We first show that $\tilde{G}$ is plus adequate.

Let $v_{1}, v_{2}$ and $c_{1}, c_{2}$ be the vertices and crossing points as illustrated in Fig. 5.7 (a). Let $e_{1}$ be the edge of $S_{+}(\tilde{G})$ illustrated in Fig. 5.7 (b).

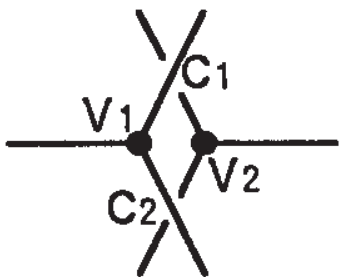

(a)

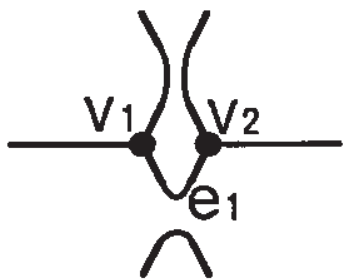

(b)

Fig. 5.7 
We note that $S_{+}(\tilde{G})-\left\{e_{1}\right\}$ is a disjoint union of circles. If $e_{1}$ is not a cut edge of $S_{+}(\tilde{G})$, then $v_{1}$ and $v_{2}$ belongs to a circle of $S_{+}(\tilde{G})-\left\{e_{1}\right\}$. Then we easily have that $c_{1}$ is a crossing of the type Fig. 5.5 (a). Therefore $e_{1}$ is a cut edge of $S_{+}(\tilde{G})$.

Similarly, if $c_{2}$ is a plus essential crossing point, then $c_{2}$ is a crossing of Fig. 5.5 (b). Therefore we have that $F_{+}(\tilde{G})$ is a graph with one edge which is not a loop and some vertices, and all plus essential crossing points join the vertices that are the ends of the edge. Suppose that there are $n$ such plus essential crossing points. By the binomial theorem we have $\sum_{k=1}^{n}(-1)^{k}\left(\begin{array}{l}n \\ k\end{array}\right)=-1$ as in section 3 . Therefore $\tilde{G}$ is plus adequate.

By the same argument we have that $\tilde{G}$ is minus adequate. Then by Corollary 4.7 and Proposition 1.3 we have $c(\tilde{G})=c(G)$.

Let $\theta\left(t_{1}, t_{2}, t_{3}\right)$ be a theta curve represented by the diagram $\tilde{\theta}\left(t_{1}, t_{2}, t_{3}\right)$ of Fig. 5.8 where $t_{1}, t_{2}$ and $t_{3}$ are non zero integers and the box of $t_{i}$ represents $\left|t_{i}\right|$ right (resp. left) handed full twists when $t_{i}>0$ (resp. $t_{i}<0$ ).

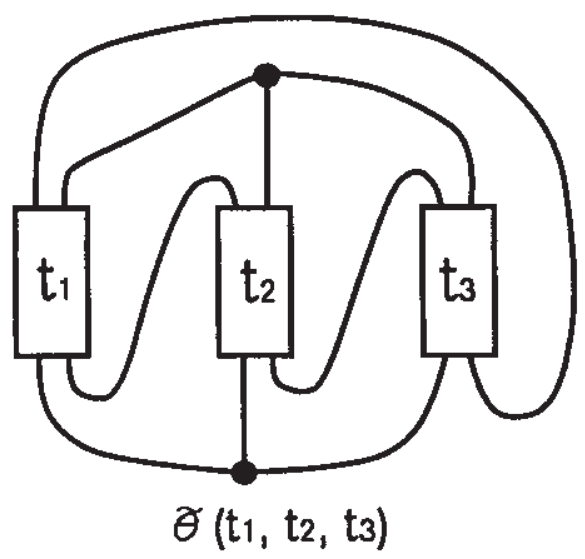

Fig. 5.8 
If all $t_{i}$ is positive or negative, then we can deform $\theta\left(t_{1}, t_{2}, t_{3}\right)$ as illustrated in Fig. 5.9.
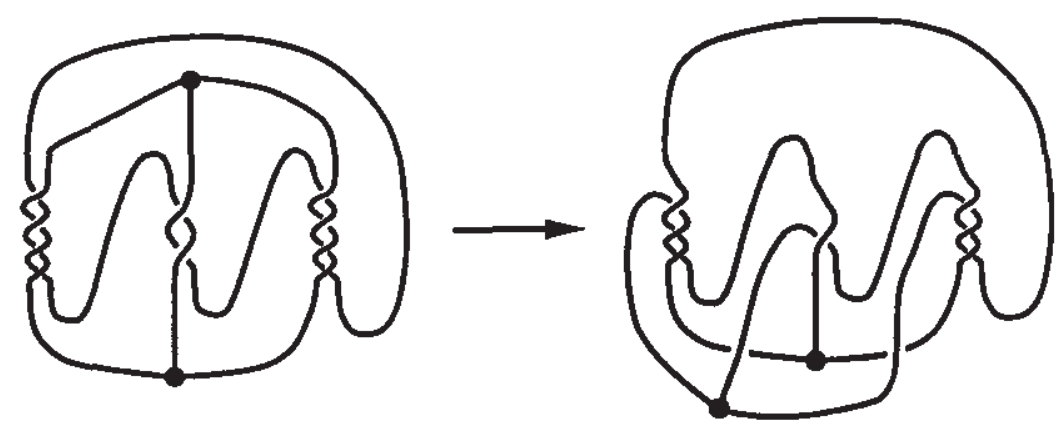

Fig. 5.9

Then we can apply Theorem 5.5 and conclude that $c\left(\theta\left(t_{1}, t_{2}, t_{3}\right)\right)=$ $c\left(\tilde{\theta}\left(t_{1}, t_{2}, t_{3}\right)\right)-1$.

If not all $t_{i}$ is positive nor negative, then we can easily check by a calculation that $\tilde{\theta}\left(t_{1}, t_{2}, t_{3}\right)$ is adequate and

$$
\beta_{1}\left(S_{+}\left(\tilde{\theta}\left(t_{1}, t_{2}, t_{3}\right)\right)\right)+\beta_{1}\left(S_{-}\left(\tilde{\theta}\left(t_{1}, t_{2}, t_{3}\right)\right)\right)=2\left|t_{1}\right|+2\left|t_{2}\right|+2\left|t_{3}\right|+1 .
$$

By Corollary $3.4, \theta\left(t_{1}, t_{2}, t_{3}\right)$ is nontrivial. But all the constituent knots of $\theta\left(t_{1}, t_{2}, t_{3}\right)$ are unknotted. Therefore $\theta\left(t_{1}, t_{2}, t_{3}\right)$ is grobally knotted and $s\left(\theta\left(t_{1}, t_{2}, t_{3}\right)\right) \leq 2$ by Proposition 5.3.

Then by substituting the equality of Theorem $3.3(3)$ to the inequality of Corollary 1.2 we have

$$
c\left(\theta\left(t_{1}, t_{2}, t_{3}\right)\right)>\frac{1}{3}\left\{6\left|t_{1}\right|+6\left|t_{i}\right|+6\left|t_{3}\right|-2\right\} .
$$


Therefore we have

$$
c\left(\theta\left(t_{1}, t_{2}, t_{3}\right)\right)=2\left|t_{1}\right|+2\left|t_{2}\right|+2\left|t_{3}\right|=c\left(\tilde{\theta}\left(t_{1}, t_{2}, t_{3}\right)\right) .
$$

\section{References}

[1] Drobotukhina, Y.V.: An analogue of the Jones polynomial for links in $R P^{3}$ and a generalization of the Kauffman-Murasugi theorem, Algebra i Analiz, 2, 1990, 171-191.

[2] Hara, M.: The Q-polynomial of the link obtained by the sum of alternating tangles, preprint.

[3] Hara, M. and Yamamoto, M.: Some links with non-adequate minimalcrossing diagrams, Math. Proc. Cambridge Philos. Soc., 111, 1992, 283-289.

[4] Harpe, P. de la, Kervaire, M. and Weber, C.: On the Jones polynomial, L'Enseign. Math., 32, 1986, 271-335.

[5] Kauffman, L.H.: State models and the Jones polynomial, Topology, $26,1987,395-407$.

[6] Kidwell, M.E.: On the degree of the Brandt-Lickorish-Millet-Ho polynomial of a link, Proc. Amer. Math. Soc., 100, 1987, 755-762.

[7] Kobayashi, K.: Reduced degree of Yamada polynomial and planarity of graphs, Science reports of Tokyo Woman's Christian University, Nos. 80-86, 1989, 963-974.

[8] Lickorish, W.B.R. and Thistlethwaite, M.B.: Some links with nontrivial polynomials and their crossing numbers, Comment. Math. Helv., $63,1988,527-539$.

[9] Miyauchi, T.: On the highest degree of absolute polynomials of alternating links, Proc. Japan Acad., 63, 1987. 174-177.

[10] Murasugi, K.: Jones polynomials and classical conjectures in knot theory, Topology, 26, 1987, 187-194.

[11] Murasugi, K.: Jones polynomials and classical conjectures in knot theory II, Math. Proc. Cambridge Philos. Soc., 102, 1987, 317-318. 
[12] Thistlethwaite, M.B.: A spanning tree expansion of the Jones polynomial, Topology, 26, 1987, 297-309.

[13] Thistlethwaite, M.B.: Kauffman's polynomial and alternating links, Topology, 27, 1988, 311-318.

[14] Thistlethwaite, M.B.: On the Kauffman polynomial of an adequate link, Invent. Math., 93, 1988, 285-296.

[15] Turaev, V.G.: A simple proof of the Murasugi and Kauffman theorems on alternating links, L'Enseign. Math. 33, 1987, 203-225.

[16] Wu, Y.Q.: Jones polynomial and the crossing number of links, Differential geometry and topology (Tianjin, 1986-87), Lecture Notes in Math., 1369, Springer, Berlin-New York, 1989, 286-288.

[17] Yamada, S.: An invariant of spatial graphs, J. Graph Theory, 13, $1989,537-551$.

[18] Yokota, Y.: The Kauffman polynomial of alternating links, preprint.

Tomoe Motohashi

NTT Telecommunication Networks Laboratories

3-9-11, Midori-cho,

Musashino-shi, Tokyo 180

JAPAN

Kouki Taniyama

Department of Matbematics

College of Arts and Sciences

Tokyo Woman's Christian University

Zempukuji: 2-6-1, Suginamiku, Tokyo 167

JAPAN
Yoshiyuki Ohyama

Department of Mathematics

Nagoya Institute of Technology

Gokiso, Showa-ku, Nagoya 466

JAPAN

Recibido: 7 de julio de 1993 